\title{
Nouvelles règles de représentativité syndicale et de négociation collective à la SNCF
}

Une réforme sous tension

New rules of union representativeness and collective bargaining at the SNCF: a

reform in a tense context

Dominique Andolfatto et Marnix Dressen

\section{(2) OpenEdition}

\section{Journals}

Édition électronique

URL : http://journals.openedition.org/travailemploi/5764

DOI : 10.4000/travailemploi.5764

ISSN : 1775-416X

Éditeur

DARES - Ministère du Travail

Édition imprimée

Date de publication : 15 septembre 2012

Pagination : 75-88

ISSN : 0224-4365

\section{Référence électronique}

Dominique Andolfatto et Marnix Dressen, « Nouvelles règles de représentativité syndicale et de négociation collective à la SNCF », Travail et Emploi [En ligne], 131 | 2012, mis en ligne le 01 décembre 2014, consulté le 01 mai 2019. URL : http://journals.openedition.org/travailemploi/5764 ; DOI :

10.4000/travailemploi.5764 


\title{
Nouvelles règles de représentativité syndicale et de négociation collective à la SNCF : une réforme sous tension
}

\author{
Dominique Andolfatto ${ }^{(*)}$, Marnix Dressen ${ }^{(* *)}$
}

\begin{abstract}
La SNCF (Société nationale des chemins de fer français) a traversé en avril 2010 un long conflit alors même qu'elle a été l'une des premières grandes entreprises françaises à mettre en xuvre la nouvelle loi sur la «démocratie sociale» qui redéfinit les règles de la représentativité syndicale et de la négociation collective. Si ce conflit a conduit à souligner les limites d'une réforme censée favoriser le dialogue, celle-ci n'en a pas moins modifié le paysage syndical caractéristique de l'entreprise ferroviaire, obligeant les acteurs des relations professionnelles à se remettre en cause et à innover.

La loi du 20 août 2008 a ouvert un nouveau contexte juridique pour les acteurs des relations professionnelles, avec pour projet une transformation volontariste de ces dernières. L'étude se focalise d'abord sur ses effets concernant la structure du jeu des acteurs au sein de la SNCF puis sur le conflit de 2010 qui illustre les difficultés du changement dans le sens attendu par le législateur et les organisations qui l'ont soutenu.

À travers cette étude de cas, l'application de cette réforme se révèle un bon analyseur des réalités du syndicalisme dans une entreprise où il compte encore beaucoup.
\end{abstract}

La loi du 20 août 2008 «portant rénovation de la démocratie sociale et réforme du temps de travail» ${ }^{(1)}$ est-elle en train de changer les relations professionnelles en France? C'est le pari et le souhait de certains acteurs sociaux et politiques parmi lesquels la Confédération générale du travail (CGT), la Confédération française démocratique du travail (CFDT), le Mouvement des entreprises de France (Medef) et la Confédération générale $\mathrm{du}$ patronat des petites et moyennes entreprises (CGPME), à l'origine de la «position commune» qui, le 9 avril 2008, jetait les bases d'une réforme adoptée l'été suivant.

La SNCF, où des élections professionnelles ont eu lieu en mars 2009, a été la première des grandes entreprises françaises concernées par le changement des règles de la représentativité syndicale et de la négociation collective introduit par la loi nouvelle. C'est pourquoi, cette entreprise de 150000 salariés

(*) Université de Bourgogne, EA Credespo; dominique. andolfatto@u-bourgogne.fr

(**) UMR CNRS Printemps / Université de Versailles-SaintQuentin-en-Yvelines; marnix.dressen@uvsq.fr

(1) Nous reprenons ici l'intitulé officiel - et double - de la loi. Mais l'analyse privilégie l'aspect «démocratie sociale» laissant de côté la question du temps de travail. Les débats sur la réforme ont néanmoins mêlé ces deux aspects, la «consolidation» des organisations syndicales - reprenant un projet de la CGT et de la CFDT - ayant été en quelque sorte «échangée» contre une plus grande décentralisation de la négociation collective (en l'occurrence sur le temps de travail) répondant aux attentes des employeurs. aux relations professionnelles complexes (et souvent conflictuelles) a été choisie comme terrain d'étude d'application de cette réforme. La SNCF a en effet éprouvé le nouveau dispositif légal tandis que ses acteurs sociaux sont en quête de nouveaux modes de régulation de leurs relations. Les organisations syndicales de la SNCF disposent également de ressources non négligeables (au titre du droit syndical ou du «statut» particulier de l'entreprise) telles des décharges de service pour activités syndicales consécutives à l'obtention du «label» représentatif et en fonction de l'audience électorale (2). Sauf à considérer que toutes les organisations en place en 2009 conservent ce «label», cette répartition des décharges syndicales (ou autres crédits horaires au profit des syndicats) va se retrouver nécessairement touchée par la réforme, ce qui redouble l'enjeu : à celui de la reconnaissance comme organisation représentative s'ajoute celui du partage des ressources.

Plus précisément, cette étude de cas ${ }^{(3)} \mathrm{s}$ 'appuie sur une vingtaine d'entretiens qualitatifs approfondis conduits avec des représentants syndicaux dans les régions Lorraine et Nord-Pas-de-Calais, ainsi que sur

(2) Selon la direction des ressources humaines (DRH) de la SNCF, le nombre de ces décharges s'élevait à 2332 équivalents temps plein en 2010. Il s'agit donc d'une ressource importante pour les syndicats. Les élections professionnelles contribuent à leur répartition (puisqu'un ratio décharges horaires / voix s'applique).

(3) La recherche de Dominique Andolfatto et Marnix Dressen a pu être menée grâce au soutien de la Dares, dans le cadre de l'enquête coordonnée par Sophie Béroud et Karel Yon (2011). 
trois entretiens avec des représentants de la direction des ressources humaines (Lorraine et nationale). L'importance des établissements ferroviaires dans les deux régions citées et, plus encore, le pluralisme marqué des équipes syndicales - avec notamment une forte présence de syndicats minoritaires, telle la CFTC (Confédération française des travailleurs chrétiens), et de solides implantations de Sud (Solidaires unitaires démocratiques) - expliquent le choix de ce terrain. Dans ces deux régions, le syndicalisme a également fait l'objet d'enquêtes sociologiques fouillées lors d'études récentes, ce qui a permis de situer les observations dans leur contexte (Andolfatto, LaBbÉ, 2007; Duriez, SaWicki, 2003; Contamin, Delacroix, 2009).

Il s'agit donc de se demander comment la réforme de 2008 a affecté les organisations syndicales. Quels changements sont intervenus dans le «dialogue social»? Les évolutions repérées s'inscriventelles dans les objectifs affichés par la loi d'août 2008? Dans cette perspective, l'étude cible tour à tour les acteurs syndicaux (et leurs recompositions éventuelles) puis leur «jeu» avec la direction qui - étonnamment (compte tenu des objectifs de la réforme de «rénovation de la démocratie sociale») et au moins provisoirement - apparaît caractérisé par un regain de conflictualité. En 2010, en effet, un long conflit va montrer combien les relations professionnelles demeurent en réalité difficiles et complexes à la SNCF avant que ne se déroulent dans un second temps, en 2011, de nouvelles élections professionnelles dont les résultats vont permettre d'ouvrir, à défaut de détendre, le jeu social.

\section{Les recompositions syndicales}

Les élections professionnelles du 26 mars 2009 ont marqué, dans le cas de la SNCF, l'entrée en vigueur des nouvelles règles de représentativité syndicale. Antérieurement, la direction de l'entreprise comptait neuf organisations syndicales : les cinq syndicats bénéficiant de la présomption irréfragable de représentativité (CGT, CFDT, FO, CFTC, CFE-CGC) ${ }^{(4)}$ et quatre organisations particulièrement développées dans l'entreprise (Unsa [ex-FMC], Sud-Rail) ou très catégorielles et spécifiques (FGAAC, SNCS) $^{(5)}$. À l'issue du scrutin de 2009, seuls quatre syndicats demeurent représentatifs au niveau de l'entreprise : la CGT, la CFDT-FGAAC

(4) FO : Force ouvrière; CFE-CGC : Confédération française de l'encadrement - Confédération générale des cadres.

(5) Unsa : Union nationale des syndicats autonomes (antérieurement : FMC [Fédération maîtrise et cadres]); Sud : Solidaires, unitaires, démocratiques; FGAAC : Fédération générale autonome des agents de conduite; SNCS : Syndicat national des cadres supérieurs. Ces organisations - non représentatives au plan national interprofessionnel - n'ont pas été associées à la négociation nationale interprofessionnelle qui a abouti à la «position commune» d'avril 2008. qui, pour la circonstance, ont entamé un processus de fusion-association, l'Unsa et Sud. Ces organisations ont recueilli au moins $10 \%$ des voix aux élections professionnelles, chiffre qui correspond désormais au seuil de représentativité. Les autres faute d'avoir atteint ce niveau - ont été ravalées au statut inférieur d'organisations non représentatives leur permettant de continuer à exister mais avec des droits réduits : elles peuvent certes désigner un représentant de section syndicale et se voir attribuer un local mais celui-ci est commun aux organisations non représentatives alors que les organisations représentatives disposent chacune de locaux spécifiques. Du moins cet état de fait est-il vrai au niveau de l'entreprise car, comme on va le voir, dans les régions ou les établissements infrarégionaux, le paysage syndical demeure plus émietté.

\section{Pas de consolidation pour les partisans de la "position commune"}

Tout d'abord, les élections de 2009 à la SNCF ne se traduisent pas par une recomposition du paysage syndical de l'entreprise autour de deux grands pôles qu'auraient constitués la CGT et la CFDT. Ces deux organisations sont en effet les principaux promoteurs - et même les seuls soutiens côté syndical - de la «position commune» du 9 avril 2008. Pour autant, elles n'en recueillent aucun bénéfice (en 2009), si ce ne sont quelques adhérents provenant d'autres organisations syndicales, dont le nombre est toutefois difficilement estimable. Bien plus, elles reculent (voir graphique 1), perdant plusieurs milliers de voix (soit des pertes nettement supérieures à la diminution du nombre d'inscrits compte tenu de restructurations récurrentes à la $\mathrm{SNCF}$ ). Le recul se révèle même sévère dans le cas de la CFDT qui, pour la circonstance, s'est alliée de façon aussi tactique qu'improbable avec la FGAAC, comme l'illustre l'encadré 1.

En 2009, l'alliance des deux organisations permet donc de sauver leur représentativité. Cependant environ un cinquième de leur électorat de 2006 ne se reconnaît pas dans cette «entreprise de représentation» et fait défaut lors du nouveau scrutin. En Lorraine, l'un des responsables régionaux de la CFDT convient d'ailleurs que probablement moins d'un électeur FGAAC sur deux de 2006 maintient sa confiance en 2009 au nouveau tandem CFDT-FGAAC. Une partie des soutiens de la FGAAC - militants ou électeurs - aurait plutôt été séduite par l'Unsa avec laquelle ils partagent une identité catégorielle ou d'entreprise. Mais si des équipes locales de l'Unsa cherchent effectivement à accueillir en leur sein des militants de la FGAAC, provoquant ainsi provisoirement la création d'organisations ad hoc comme un petit SAR (Syndicat autonomes des roulants) en Lorraine, l'Unsa nationale ne paraît pas intéressée par un tel rapprochement. Est-elle dubitative face aux résultats potentiels d'une telle alliance? Craint-elle des surenchères de la part de la FGAAC? Les cadres 
adhérents de l'Unsa éprouvent-ils l'aversion que l'on dit pour les conducteurs de trains et leur efficace pouvoir de blocage des trafics? À moins que, plus simplement, les parties en cause n'aient pas su se rencontrer comme l'affirme un ex-leader national de la FGAAC. On peut risquer une troisième hypothèse : la CFDT, l'Unsa et la direction de la SNCF n'auraient alors guère d'intérêt à ce dernier rapprochement. Si une alliance Unsa-FGAAC était intervenue, la CFDT aurait risqué de ne pas atteindre le seuil de $10 \%$ des voix. Elle aurait dès lors perdu sa représentativité de sorte que le projet de la direction de la SNCF de conserver un «pôle réformiste» à la hauteur d'au moins $30 \%$ d'audience pour être en capacité de signer des accords, s'évanouissait. À elle toute seule, l'Unsa ne pouvait atteindre ce seuil, compte tenu de l'élimination prévisible des autres syndicats «réformistes» (FO, CFTC, CFE-CGC).

\section{Encadré 1 \\ L'alliance CFDT-FGAAC}

Cette alliance entre le syndicalisme plutôt général et de type universaliste de la CFDT et un type de syndicalisme catégoriel des conducteurs de train perdure contre toute attente. Elle a été dictée par la fragilité de l'audience de la CFDT qui a fortement reculé lors des élections de 2004, à la suite de sa prise de position lors de la réforme des retraites des fonctionnaires l'année précédente, même si les cheminots ne sont pas des fonctionnaires mais, pour la plupart, agents «à statut». Quant à la FGAAC - qui a pour unique objectif de se consacrer aux 15000 conducteurs - elle était assurée de perdre sa représentativité au niveau de l'entreprise où, à elle seule, elle ne pesait que moins de $3 \%$ des cheminots toutes catégories confondues au moment de la réforme ${ }^{(1)}$. Malgré des identités très différentes, ces deux organisations ont donc décidé de s'associer. Elles sont parvenues au compromis suivant : la FGAAC devient la branche « conducteurs de trains" de la CFDT cheminote. Pour autant, dans le ferroviaire privé, la FGAAC a longtemps refusé cette sorte de "fusion acquisition» pour préserver son indépendance. Aujourd'hui, cette époque est en principe révolue mais, curieusement, la FGAAC continue à siéger ès qualités, indépendamment de la CFDT, dans le long processus de négociation d'une convention collective encore en cours. II arrive que tel ou tel accord de branche, partie de la future convention collective, soit signé par une organisation et pas par l'autre.

(1) Contrairement aux journalistes et aux pilotes de ligne, la FGAAC n'a pas cherché à se faire reconnaître comme syndicat dont la représentativité serait calculée au niveau d'un collège spécifique et non d'une entreprise tout entière. Faute de réseaux d'influence - sinon d'un savoir-faire en termes de lobbying - aussi forts que ceux des deux professions précédentes, il est d'ailleurs loin d'être assuré qu'elle aurait obtenu gain de cause.

Finalement, au prix de son alliance avec une organisation syndicale qui ne lui ressemble pas, la
CFDT conserve de justesse, sa «représentativité». Cependant son audience recule en 2009 et si celle de l'Unsa progresse, ce «pôle réformiste» (CFDTFGAAC d'une part, et Unsa d'autre part) n'atteint pas le seuil des $30 \%$. Enfin, si la CFDT réussit à préserver sa représentativité, nos observations montrent que ce recul induit un certain stress dans l'organisation. On pourrait y voir une canalisation des énergies militantes, l'obligation à plus de discipline interne qui, au fond, contribue, elle aussi, à changer la culture syndicale.

\section{Graphique 1 : Évolution des audiences syndicales réelles à la $\mathrm{SNCF}$ (en \% des électeurs exprimés)}

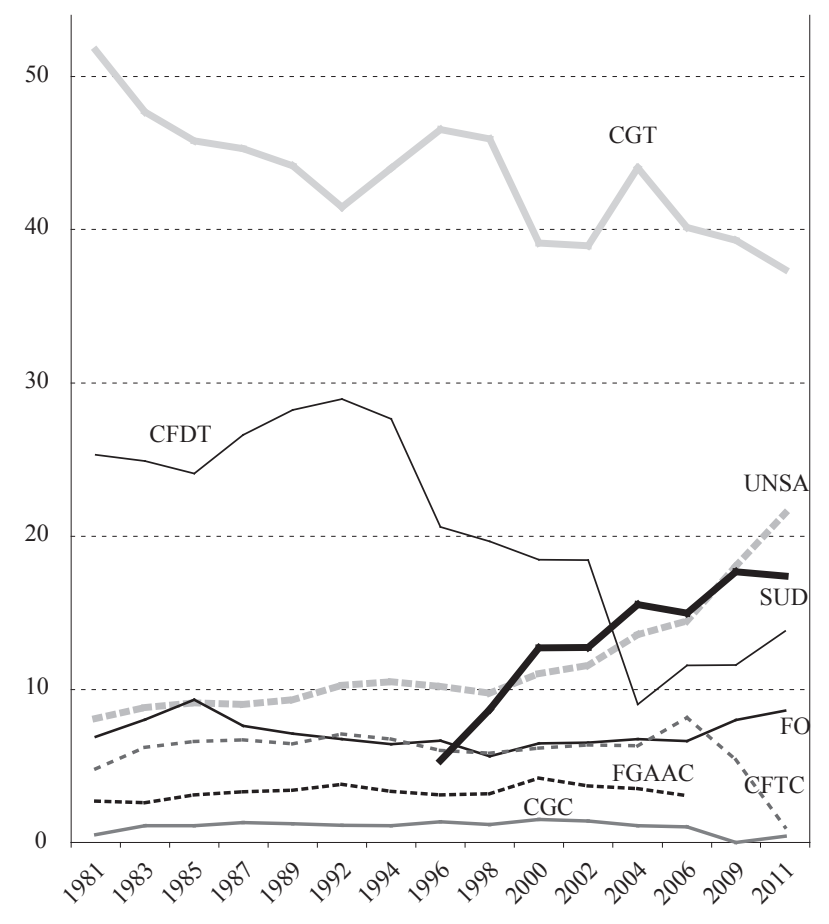

Source : SNCF, DRH. Graphique réalisé par nos soins.

\section{Effet d'aubaine pour l'Unsa et pour Sud-Rail?}

En 2009, à la différence de la CGT et de la CFDT, l'Unsa et Sud progressent assez sensiblement lors des élections professionnelles en pourcentages mais aussi en nombre de voix, ce qui est d'autant plus remarquable que le nombre des inscrits a reculé et que l'abstention augmente. Or, ces organisations n'ont pas été associées à la négociation nationale qui a abouti à la «position commune» que l'Unsa notamment avait rejetée pour cette raison

L'Unsa - nationale - estimait même que la «position commune» allait produire des «conséquences... désastreuses» : «difficulté de créer un syndicat, [...] [moins] de liberté pour adhérer au syndicat de son choix [...], montée de syndicats ultra-catégoriels $[\ldots]$ et explosion des coordinations qui ne se reconnaîtront pas dans les deux syndicats "officiels"»; autrement dit, elle en dressait un tableau apocalyptique. Naturellement, elle voyait 
la question des seuils de représentativité et, plus encore, le délai de deux ans imposé pour qu'un syndicat non affilié à une confédération représentative soit reconnu au plan national comme une façon de la marginaliser. À ses yeux, ce dispositif devait conduire à « un hold-up organisé sur la démocratie» de la part des signataires de la «position commune» ${ }^{(6)}$.

À la SNCF, ces sombres prévisions ne se sont donc pas réalisées. L'Unsa voit même ses positions consolidées. Ses gains, lors des élections professionnelles de 2009, proviendraient notamment d'anciens adhérents de la FGAAC qui ont refusé de rejoindre la CFDT. En Lorraine, un responsable cédétiste évalue à environ $25 \%$ les soutiens de la FGAAC ralliés à l'Unsa.

En 2008, l'Union syndicale solidaires (USS, regroupant les syndicats Sud) faisait une lecture de la «position commune» tout aussi critique(7). Elle estimait que la période de deux ans imposée aux nouveaux entrants «représente un frein à l'activité syndicale». Si Sud et l'Unsa n'étaient pas favorables au statu quo ante, la "position commune» leur semblait favoriser un duopole qui ne pourrait se constituer qu'au détriment des syndicats les moins influents. Or, à la SNCF, ces craintes se sont finalement révélées sans fondement.

En somme, dans l'entreprise ferroviaire, ces premières élections ont paradoxalement traduit le succès d'opposants à la réforme tandis que les organisations qui l'ont soutenue n'engrangeaient aucun bénéfice.

Un rapide examen des faits pourrait conduire à parler d'un effet d'aubaine pour les opposants à la «position commune». Cependant une étude plus dialectique de la situation tend à montrer que le renforcement de l'Unsa et de Sud ne résulte pas essentiellement d'une causalité juridique (la loi), tandis que celui de l'Unsa seule ne procède pas uniquement du déclin du "pôle réformiste» traditionnel et celui de Sud du déclin de la CGT; il ne s'agit pas non plus purement des fruits d'un travail d'équipes syndicales, de leurs valeurs ou de leurs identités professionnelles, mais d'une combinaison des trois facteurs.

\section{Quel devenir pour FO, la CFTC et la CFE-CGC?}

Les organisations éliminées - au niveau national - n'ont pas nécessairement démérité sur le plan électoral : FO et la CFE-CGC, qui font liste commune en 2009, malgré, là aussi, de profondes différences idéologiques et culturelles conservent le

(6) Déclaration confédérale, UNSA magazine, mai 2008.

(7) Communiqué de l'USS, 10 avril 2008. même nombre de voix qu'en $2006^{(8)}$. Cependant, en dehors des exceptions régionales, ces organisations perdent leur représentativité nationale. Il en découle que leurs représentants sortants doivent abandonner les différents mandats ou décharges horaires dont ils bénéficiaient ${ }^{(9)}$. Cela oblige parfois ces élus, voire ces «permanents», à retourner occuper un poste de travail classique. Cette situation est souvent vécue douloureusement, comme une sorte d'échec personnel, nourrissant amertume et regard critique sur une réforme qui sanctionne des syndicalistes jusque là fortement impliqués dans le système des relations professionnelles.

La perte de représentativité est encore plus problématique pour la CFTC en raison de l'éclatement de nombre de ses équipes, de déchirements internes ayant parfois pris un tour dramatique, et d'une stratégie devenue hésitante de ce fait. Sur le terrain, pour sauver leurs mandats, certains de ses responsables préfèrent rejoindre d'autres organisations. Tous les cas de figure se produisent et les confusions-recompositions se poursuivent aux lendemains des élections dans la perspective du scrutin de 2011. La CFTC se refuse à un rapprochement avec la CFDT qui aurait pourtant pu paraître logique. Au contraire, tout se passe comme si la «déconfessionnalisation» (10) de 1964 demeurait un traumatisme pour des militants qui ne l'ont pourtant pas vécue (ce qui interroge sur la fabrication des identités syndicales et l'impact d'événements historiques) ${ }^{(11)}$. Existe également un ressentiment à l'égard d'une organisation à l'origine de la «position commune» fustigée comme la cause des difficultés actuelles et, peut-être à terme, de la mort de la CFTC. Enfin, l'approche de la négociation par les deux organisations n'est pas de même nature. En Lorraine, l'un des responsables de la CFTC reproche à la CFDT un jeu «tordu» et se déclare favorable à la construction de rapports de force. De façon étonnante, la CFTC - toujours en Lorraine se trouve plus d'affinités avec FO, pourtant parfois très fortement laïque, qu'avec la CFDT ou d'autres organisations.

Dans la perspective des élections de 2011, FO, la CFE-CGC et la CFTC tentent de faire cause commune. De ce point de vue, la loi favoriserait bien un regroupement mais à la $\mathrm{SNCF}$, ce dernier,

(8) FO regroupe en son sein des courants allant de la droite de l'échiquier politique à la gauche radicale d'inspiration trotskiste. Cela explique certaines fractures internes et notamment qu'à la SNCF, le choix de l'alliance avec la CFE-CGC, au nom d'une certaine ligne réformiste ou réaliste, ait peiné à convaincre la «base».

(9) Voir la note $n^{\circ} 2$, supra.

(10) La CFTC s'était alors transformée en une organisation laïque, la CFDT, ce que refusa une petite partie de la CFTC dite un temps «CFTC maintenue».

(11) Dans ce registre, voir BoIs (1971) : l'auteur attribue certains comportements électoraux contemporains à des traumatismes historiques remontant à l'époque révolutionnaire. 
même circonstanciel, peine à prendre forme et, dans ce cas précis, n'aboutit finalement pas.

\section{Une « resyndicalisation" qui se fait attendre?}

En plus de recomposer et simplifier le paysage syndical, la loi d'août 2008 est censée favoriser la resyndicalisation et donc inverser l'évolution du taux de syndicalisation depuis les années 1970 (même si son déclin s'est nettement ralenti depuis quelques années, comme si un plancher avait été atteint; Andolfatto, LabBÉ, 2009). La représentativité étant désormais assise sur le vote, les promoteurs de la réforme ont postulé que celle-ci obligerait à sortir d'un syndicalisme d'appareils ou «professionnalisé». Avec la réforme, les syndicats devraient à nouveau «cultiver» leur base, recruter de nouveaux adhérents, consolider leurs implantations pour recueillir ensuite des voix et assurer leur pérennité. Pour autant, malgré la mise en œuvre de la loi de 2008, aucune resyndicalisation ne semble en vue selon les différents responsables syndicaux rencontrés. Une étude d'Entreprise \& personnel le confirme plus globalement et indique que les «élus des salariés» tireraient, en réalité, de moins en moins leur force du nombre des adhérents et des militants malgré la loi de 2008, mais évolueraient vers un rôle de «lanceur d'alerte» (au sein du comité d'hygiène, de sécurité et des conditions de travail [CHSCT] notamment) dont l'objectif est d'attirer l'attention en interne ou en externe sur certains risques (pour la santé des salariés ou l'environnement par exemple) ou sur des pratiques jugées problématiques ${ }^{(12)}$.

En outre, selon notre enquête, les dissensions entre organisations perdurent, ce qui n'encourage sûrement pas la syndicalisation. De plus, le seul regroupement entre structures syndicales qui soit intervenu à la SNCF a concerné la CFDT et la FGAAC et n'a pas constitué la force d'attraction espérée. Les électeurs de cet attelage se révèlent en 2009 moins nombreux que la somme des suffrages réunis par ces deux organisations antérieurement à leur rapprochement.

Toutes les organisations demeurent donc confrontées à une même problématique : celle du renouvellement de leurs effectifs, compte tenu notamment d'un «papy boom syndical», autrement dit le départ à la retraite d'importantes cohortes de militants. Or ces derniers sont remplacés avec difficultés par des plus jeunes, malgré la mise en œuvre de politiques ad hoc de recrutement et l'utilisation à la SNCF de "chèques syndicaux» (dits $\mathrm{AY}$ ) qui

(12) Voir Dubreuil, Basilien (2012) : selon les auteurs de cette étude, la réforme de 2008 est perçue dans les entreprises comme ayant fait symboliquement évoluer le statut des représentants du personnel (pp. 18-19), sans pour autant avoir de retombées réelles sur les dynamiques internes de l'entreprise. Quant à la «démocratie sociale», elle n'est jamais évoquée et resterait considérée comme une utopie ou un tabou. permettent l'organisation de sessions de formation «découverte du syndicalisme». Une partie de ces crédits de formation syndicale attribués par la direction de la SNCF aux syndicats est redistribuée aux salariés afin de les associer à ces formations qui se déroulent sur le temps de travail et qui peuvent être l'occasion de détecter certains «potentiels».

Il conviendrait aussi d'évoquer un «syndicalisme de service» dont il est difficile d'apprécier l'attraction sur de nouveaux adhérents. Plusieurs syndicats de cheminots sont en effet en concurrence pour offrir à leurs contacts un «pack» d'adhésion comprenant au moins un abonnement téléphonique à un prix très avantageux, une assistance juridique, les services d'une association de consommateurs, un capital versé en cas d'accidents graves du travail, etc.

La situation est encore plus problématique pour les organisations qui ont perdu leur représentativité. Elles s'efforcent tant bien que mal de faire vivre un réseau lorsque celui-ci existait comme dans le cas de la CFTC, en Lorraine par exemple, ou de FO. Elles maintiennent donc des sections syndicales et tentent de prendre appui sur la nouvelle disposition de la loi de 2008 - le représentant de la section syndicale (RSS) - pour demeurer un interlocuteur, certes secondaire, du management. Mais cette institution peut être perçue également comme une régression, l'acception de ce qui ne serait qu'un sous-statut. D'une organisation à l'autre, les usages des RSS se révèlent variés.

En Lorraine, la CFDT, qui avait perdu sa représentativité régionale à la suite du scrutin de 2009, voit dans le RSS une sorte de refuge provisoire, une institution-relais, qui permet de conserver des ressources et de demeurer un acteur des relations professionnelles. Cette attitude se trouve facilitée par la bienveillance que la direction maintient envers la CFDT là où elle a perdu sa représentativité, d'abord parce que la CFDT-FGAAC a conservé sa représentativité au niveau national de l'entreprise mais aussi parce qu'elle demeure un appui indispensable pour construire ce «pôle réformiste» que la direction ne désespère pas de faire émerger.

A contrario, d'autres organisations comme la CFTC et FO refusent de jouer ce jeu de la loyauté («loyalty») propre à ce nouveau dispositif légal. Elles préfèrent sortir du jeu («exit»), protester («voice») et, en l'occurrence, tenter de s'appuyer sur d'autres ressources pour compenser la perte de la «représentativité» (HiRsChMAN, 1970). Il est vrai que ces mêmes syndicats - qui n'ont conservé leur représentativité que dans quelques régions ou établissements de la SNCF - ne bénéficient plus de la même sollicitude de la part de la direction que la CFDT.

Les militants FO interviewés sont amers et disent préférer «retourner sur le terrain» et reprendre un 
poste de travail classique puisqu'ils ont perdu leurs mandats. La CFTC-Lorraine, plus influente qu'au niveau national, a au contraire privilégié l'action judiciaire pour contester les conséquences de la mise en œuvre de la loi nouvelle concernant, par exemple, le fonctionnement des comités d'entreprise. Elle se plaint notamment d'être victime de discrimination de la part des autres syndicats et de l'employeur. Plus fondamentalement, la défense du pluralisme syndical, le refus de faire du suffrage professionnel le seul étalon de la représentativité, ce qui évoque des conceptions anciennes du mouvement ouvrier(13), sont au nombre des principaux arguments opposés à la loi nouvelle.

\section{Le reclassement des élus et permanents}

Si la réforme n'a donc pas produit la recomposition et la resyndicalisation attendues, on observe que sont intervenus des transferts de militants qui disposaient de mandats représentatifs et qui, bénéficiant d'importantes décharges horaires, étaient souvent devenus des militants professionnels ou des permanents. Les représentants appartenant aux organisations les plus menacées par la loi de 2008 sont parfois allés proposer leurs services et apporter leurs soutiens à d'autres syndicats assurés de demeurer dans le jeu. Autrement dit, dans certains cas, les recompositions n'ont pas eu lieu au niveau des organisations mais de certains individus.

À la SNCF, deux vagues de transferts se sont produites : en amont des élections de mars 2009 puis dans la perspective du scrutin de 2011. La première vague a vu, par exemple, de nombreux permanents CFTC migrer vers la CFDT, notamment dans la région lyonnaise. En Lorraine, le président $\mathrm{du}$ secteur régional de la fédération des cheminots affirme avoir préféré rejoindre la CGT avec une centaine d'adhérents. Dans un tract non daté diffusé début 2010, il explique sa démarche par refus «des alliances contre-nature avec la CFE-CGC et FO» que projette la CFTC pour recouvrer sa représentativité. Il indique aussi qu'il a évalué les différents «choix » qui s'offraient à lui. Il n'a pas choisi l'Unsa qui «reste toujours un allié objectif de la direction», ni Sud «essentiellement protestataire» et non adossé à une confédération, ce qui présenterait un «risque» d'affaiblissement à terme, ni la CFDT qui «accompagne le libéralisme» et qui, par «son alliance avec la FGAAC favorise l'action corporatiste des agents de conduites». Il s'est donc tourné vers la CGT, «syndicat contestataire et constructif» dont il apprécie aussi la stratégie de rassemblement au niveau confédéral. Il ajoute encore qu'il a passé «des bons comme des mauvais moments» à la

(13) Selon un argument ancien, «la représentativité dérive d'un présupposé sociologique - l'homogénéité du groupe de production - et non d'une arithmétique liée à un quelconque suffrage : elle est un donné social et non un constat politique» (RosanVallon, 1987, pp. 208-209).
CFTC, qu'il a «apprécié certains militants pour leur travail et leur dévouement... mais [les a] détesté [s] quand leur intérêt personnel passait avant l'intérêt collectif.» Dans le Nord, ce sont des responsables du syndicat CFTC qui ont rejoint Sud, pour des raisons différentes selon les individus : jeune militant combatif, trésorier du syndicat chrétien ou cadre autodidacte en fin de carrière, peu motivé par un retour à un poste de travail.

Pour décrire ces transferts, au moment de notre enquête en Lorraine, les syndicalistes recouraient parfois avec ironie au vocabulaire footballistique : le «mercato». Il existerait ainsi une sorte de marché des permanents qui, quelle que soit leur étiquette d'origine, se «vendraient» au syndicat le plus offrant, en l'occurrence celui qui leur permettra de conserver leur(s) mandat(s) et leur statut de «permanent», dit «agent en service libre» à la SNCF, en échange de l'apport des électeurs qu'ils promettent d'amener avec eux.

On peut par conséquence s'interroger sur les influences syndicales. Les étiquettes syndicales seraient finalement moins décisives que les candidats qui, une fois élus, deviendraient des «notables locaux» - pour emprunter une expression de la sociologie du système politique local (MABILEAU, 1991) - disposant d'une clientèle fidèle et cherchant à faire prospérer leur «entreprise de représentation». Sans doute faut-il se garder de généralisation hâtive mais ces transferts posent la question des identités syndicales qui s'effaceraient derrière des ambitions individuelles. Ceux qui restent fidèles à leur organisation d'origine, malgré la perte éventuelle de la représentativité, décrivent ces transferts comme le fait de «mercenaires », s'engageant là où les conditions d'un militantisme professionnel sont les plus pérennes ${ }^{(14)}$.

Le défi de la syndicalisation demeurerait donc entier. Dans une analyse à chaud de la réforme de 2008, Antoine BEVORT (2008) signalait à ce propos : «En l'absence de tout avantage pour l'adhérent, l'épuisement d'une forme syndicale reposant sur l'implication forte des adhérents (sur une identification autant idéologique et militante que sociologique) et son remplacement par un modèle politique de représentation reposant sur une compétition organisationnelle et sur la délégation plus que sur la représentation de la profession et sur le mandatement, décourage l'adhésion».

(14) Ces transferts peuvent aussi renvoyer à l'attachement des délégués et des élus syndicaux à leur fonction de représentation et de défense des salariés, qui primerait sur une orientation syndicale qui peut tenir au contexte local (syndicats présents, personnes exerçant les mandats) autant qu'au programme revendicatif de telle ou telle organisation. 


\section{Les difficultés persistantes du "dialogue social"}

En obligeant à une re-légitimation des acteurs syndicaux par le suffrage professionnel et à la constitution de majorité d'engagement pour la signature d'accords, la loi du 20 août 2008 vise officiellement la «rénovation de la démocratie sociale». À la SNCF, la réforme a été bien accueillie par l'employeur comme par les organisations syndicales qui ont conservé leur représentativité. Beaucoup évoquent des séances de négociations qui auraient gagné en fluidité et qui ne s'ouvriraient plus par la lecture d'une dizaine de déclarations liminaires. On entrerait plus vite dans le vif du sujet. «Avec moins de gens autour de la table» selon un interlocuteur syndical mais aussi un représentant de la direction, la discussion serait plus féconde. Les organisations qui demeurent représentatives auraient également gagné en légitimité. Pour autant, ce nouveau contexte juridique n'a pas empêché l'une des plus longues grèves que la SNCF ait connues depuis ces dernières années. Et ce conflit a souligné combien simplement se parler et négocier demeuraient difficiles.

Par ailleurs, le paysage syndical à la $\mathrm{SNCF}$ - et donc les acteurs possibles du dialogue - apparaît désormais à géométrie variable, ce qui peut poser des problèmes d'articulation entre les différents niveaux de relations professionnelles. En effet, à la $\mathrm{SNCF}$, la représentativité des organisations est mesurée au niveau de vingt-sept comités d'établissements et c'est l'agrégation des résultats de ces vingt-sept élections qui donne la liste des organisations représentatives au niveau national de l'entreprise. Or la liste des organisations reconnues représentatives au niveau du pays tout entier n'est pas nécessairement identique aux situations régionales (voir tableau en annexe). En descendant au niveau des établissements infrarégionaux, on observe encore d'autres différences. Par exemple à la suite des élections professionnelles de 2009, la CFDT quoique représentative au plan national, ne l'est pas dans la région Champagne-Ardenne tout en étant majoritaire dans l'un des établissements de cette même région. De plus, une organisation peut éventuellement être majoritaire au niveau des élections des délégués du personnel et minoritaire à l'élection du comité d'entreprise. Cette hétérogénéité dans la représentativité ne constituerait-elle pas un véritable casse-tête juridique? Pour les gestionnaires des ressources humaines, cela semble bien être le cas et il est donc difficile dans ces conditions de parler d'une simplification du paysage syndical et d'une facilitation du «dialogue social». De plus, les organisations syndicales non représentatives peuvent créer des sections et désigner des RSS et, par conséquent, demeurer des acteurs certes secondaires, mais bien présents, des relations sociales. Au-delà de ces aspects juridiques, la grève demeure également une caractéristique centrale de l'entreprise et le dernier conflit important qui l'a marquée n'est manifestement pas sans lien avec certains chamboulements intervenus dans les relations sociales après la mise en œuvre de la loi de 2008. À tout le moins, une période d'apprentissage aux nouveaux rapports institués par cette loi a été nécessaire et a impliqué - indirectement - un conflit avant que ne soit trouvé un nouvel équilibre - certes provisoire -, après les élections professionnelles de 2011.

\section{Le conflit comme mode de régulation}

Si les évolutions du paysage syndical de la SNCF après les élections de 2009 semblent peu perceptibles par les salariés des différentes catégories, des changements, plus ou moins contraints ou latents, sont bel et bien en cours. Pour autant, il reste illusoire de mesurer les effets de la loi sans replacer son application sur la toile de fond des restructurations permanentes de la SNCF (redéfinition des périmètres des établissements, filialisation), qui brouillent les effets de la loi et en complexifient l'application et l'interprétation dans cet établissement public industriel et commercial. Le nouveau cadre juridique conjugué à une conjoncture politico-économique incertaine incite les syndicats et les managers à un jeu plus tactique encore que par le passé.

Du 7 au 21 avril 2010, la SNCF va ainsi connaitre un long conflit ouvert qui montre combien les relations professionnelles peuvent y demeurer problématiques d'autant plus qu'il apparaît même échapper à la compréhension des acteurs, ce qui n'est pas la première fois dans le secteur ferroviaire. En cela, il interroge finalement un certain mode de relations professionnelles (VACQUIN, 2009) et constitue une raison supplémentaire pour tenter d'en dégager quelques traits en examinant ses liens avec la réforme de la représentation syndicale.

\section{Un conflit aux causes multiples}

Officiellement le conflit d'avril 2010 s'appuie sur un long cahier de revendications : emploi, avenir de l'entreprise, avenir du fret, ouverture de la concurrence dans les transports régionaux, salaires. Une si longue liste peut s'interpréter comme le signe de frustrations aussi nombreuses que diffuses. De fait, ce conflit renvoie à plusieurs causes profondes.

Il résulte d'abord d'inquiétudes engendrées par d'importantes restructurations (concernant notamment le fret) et la filialisation croissante d'activités. La SNCF est un établissement public, qui contrôle une holding de 48 entités et de 650 filiales, dont certaines sont en concurrence directe avec elle. Certes la SNCF compte encore 150000 salariés mais le nombre de ceux qui relèvent du groupe sera 
bientôt plus important que ceux qui émargeront à la maison mère et conserveront le «statut» de cheminots. Dans un avenir proche, si la tendance actuelle se poursuit, la plus grande partie du chiffre d'affaires de la SNCF émanera du groupe et non plus de l'établissement public. Ce qu'il faut bien appeler une «privatisation rampante» ${ }^{(15)}$ correspond à la volonté de la direction de contourner les résistances des cheminots pour mieux préparer la SNCF à l'ouverture progressive à la concurrence du marché du transport ferroviaire, voulue par la Commission européenne et relancée de manière plus volontariste depuis 1991. Or, nombre de cheminots, par attachement au service public ou par crainte de perdre un statut encore protecteur, n'adhèrent que faiblement aux orientations de l'entreprise qu'ils ne comprennent d'ailleurs pas toujours tant elles apparaissent à beaucoup d'entre eux incertaines et chaotiques. De plus, ces réorientations ne portent guère leurs fruits puisque chaque semaine apporte son lot de nouvelles souvent perçues comme des menaces : progression du fret privé, transformation de la SNCF en société anonyme, dégradation de sa notation financière, projet d'un «cadre social harmonisé» commun à tous les cheminots de France, transfert des cheminots SNCF vers des opérateurs privés si ces derniers remportent les appels d'offres au niveau des régions, pénétration de la concurrence dans le transport voyageur. Comme leurs organisations syndicales, les cheminots ont aussi le sentiment de se heurter à une intransigeance croissante de la direction de la SNCF et du pouvoir politique qui paraît encourager ce raidissement. Ce dernier affiche sa détermination à balayer les freins à la «modernisation» des relations sociales en général et au sein de la SNCF en particulier, et à assouplir les résistances des syndicats, notamment celles de la CGT et de Sud, réputés arc-boutés sur la défense «d'archaïsmes», qui plus est «corporatistes» et «égoïstes» selon des stéréotypes que l'on peut juger éculés.

\section{La montée des tensions}

Le conflit d'avril 2010 n'éclate pas comme un coup de tonnerre dans un ciel serein ${ }^{(16)}$. Il est précédé par le demi-échec de deux journées d'action dites «carrées » (c'est-à-dire non reconductibles) organisées en janvier puis en février 2010 et ciblant notamment les restructurations. Échec aussi en mars de la négociation sur les salaires lors de laquelle la direction de la SNCF, comme elle le fait depuis plusieurs années, avait conditionné une «augmentation générale» de $1,3 \%$ à la signature des syndicats et prévenu que, faute d'accord, la

(15) Selon l'expression déjà ancienne employée par Pierre Dubois (1974) dans Mort de l'État-patron.

(16) Pour une perspective diachronique sur près d'un quart de siècle des conflits dans le transport ferroviaire en France, voir Andolfatto, Dressen, Finez, 2012. hausse ne serait que de $0,9 \%{ }^{(17)}$. Mentionnons aussi le résultat mitigé de la journée d'action interprofessionnelle du 23 mars 2010. Le contexte immédiat de la grève, c'est aussi le mécontentement grandissant de militants CGT qui ne se satisfont plus de rituelles journées d'actions sans résultat. D'après Les Échos du 22 avril 2010, à Toulouse, Marseille, Montpellier et Clermont-Ferrand, des adhérents de la CGT auraient rejoint Sud, organisation perçue comme plus déterminée à s'engager dans une lutte ouverte et franche. En outre, la CGT n'apprécie pas que le président de la SNCF cherche à isoler son syndicat en travaillant à la construction d'un «pôle réformiste» fondé sur une alliance privilégiée avec l'Unsa et la CFDT-FGAAC, alors qu'à la suite des élections de 2009, ces dernières ne peuvent signer seules le moindre accord, même en additionnant leur audience qui demeure inférieure à $30 \%$.

En fait, la CGT paraît s'engager dans ce conflit surtout parce qu'elle subit une concurrence menaçante de Sud. En outre, malgré son score très honorable aux élections de mars 2009 (presque $40 \%$ des suffrages exprimés), elle ne parvient pas à être considérée par la direction comme susceptible de jouer un rôle plus «responsable» ou plus «engagé» dans les relations sociales (dans le sillage de la CGT d'Électricité de France [EDF], pour risquer une comparaison). Il est vrai que la CGT a du mal à intégrer ce rôle, une partie de ses militants restant attachés à la lutte des classes et s'opposant à toute remise en cause du statut du cheminot, encore protecteur pour le personnel si on le compare aux accords de branche conclus dans le ferroviaire privé (Cohen, Dressen, 2011). Pour simplifier, le dilemme de la CGT peut se formuler ainsi : comment recourir moins systématiquement au rapport de force sans céder à Sud qui représente l'aile gauche de l'échiquier syndical?

L'ambition d'occuper tout l'espace n'est pas sans lien avec la mise en œuvre de la loi de 2008. Les nouvelles règles de représentativité syndicale et de régulation des relations professionnelles qui en découlent placent en effet la CGT des cheminots «au centre du jeu» (ainsi que l'indique Didier Le Reste ${ }^{(18)}$, son secrétaire général, au lendemain des élections professionnelles de mars 2009). Ce faisant, la CGT signifie qu'il faut compter avec elle, quitte à renouer avec un système parfois qualifié de «pacte micro-corporatiste» (JOBERT, 1996; TEXIER, 1998) ou de «cogestion» direction-CGT (comme à EDF).

(17) Ce qui sera finalement le cas. Pourtant, rien n'interdisait à la direction de décider unilatéralement une augmentation des salaires de $1,3 \%$. Ce choix participe aussi du rapport de force avec les syndicats, voire d'un type de relations qui rappelle «l'univers militaro-hiérarchique de la SNCF» dont parlait Georges RiBEILL (1994).

(18) Cité par Fabrice Amedeo, «La CGT seul maître à bord», Le Figaro, 27 mars 2009. 
Le graphique 2 montre que la CGT a plutôt joué un rôle d'opposant dans la période antérieure à la réforme. Avec FO et Sud, elle apparaît en effet comme l'un des syndicats avalisant le moins d'accords quoique cette opposition ne soit guère systématique. La loi de 2008 lui confère de nouvelles responsabilités dans la négociation qu'elle souhaite mettre à profit. Pour autant, l'organisation paraît prise entre deux feux : sur sa gauche, Sud la surveille et, sur sa droite, le «pôle réformiste» cherche à demeurer l'interlocuteur privilégié de la direction.

Graphique 2 : Taux de signature des accords par les syndicats à la SNCF, 2005-2009, avant l'application de la loi de 2008 en \% des accords

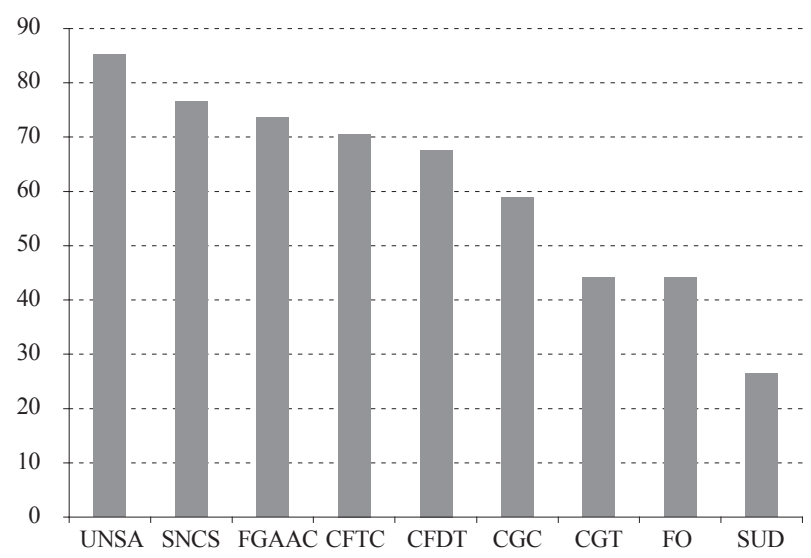

Source : SNCF, DRH. Graphique réalisé par nos soins.

$N B$ : Au cours de cette période, 34 accords ont été signés.

\section{Un conflit ouvert : la CGT deux fois prise en tenaille}

Le graphique 3 donne à voir l'évolution de la participation des cheminots au fil du long conflit du printemps 2010 (dont plus de quinze jours de grève reconductible) et, notamment, la participation des conducteurs de train (les ADC [agents de conduite]) et, plus encore, celle des contrôleurs (les ASCT [agents du service commercial trains]), en demande de reconnaissance compte tenu des transformations de leur métier. Ce conflit peut être découpé en trois périodes.

Entre le 23 mars et le 6 avril 2010, la CGT tente de faire d'une pierre deux coups en essayant de dénouer les liens privilégiés entre le «pôle réformiste» et le management de la SNCF et d'isoler Sud-Rail, son plus proche concurrent sur le plan idéologique et stratégique. Dans la poursuite de ce double objectif, la direction de la CGT des cheminots développe une politique d'unité d'action exclusive avec l'Unsa et la CFDT-FGAAC. Pour le 7 avril, sept préavis de grèves catégorielles et reconductibles sont déposés par la CGT. Pour le 8, un autre préavis concerne tous les cheminots, mais pour une grève "carrée» de 24 heures. «Ce qu'imaginait la CGT, c'est que le 9 au matin, la direction allait être obligée de céder pour un retour à la normale avant le week-end des vacanciers» commente un syndicaliste (Les Échos,
22 avril 2010). Mais le "pôle réformiste» se refuse à jouer le jeu. L'Unsa entend préserver ses forces contre la réforme, plus globale, des retraites qui se profile tandis que la CFDT-FGAAC se désengage après avoir obtenu satisfaction sur quelques revendications, notamment salariales. La grève sera donc l'affaire de la CGT et de Sud.

Du 6 avril, premier jour de grève, au 20 avril, le «pôle radical» se retrouve en première ligne. Avec le retrait des réformistes, la CGT est confrontée à la situation qu'elle voulait éviter : un coude-à-coude, sinon un face-à-face, avec Sud. Pire, la CGT est cette fois prise en tenailles entre Sud, qui a déposé ses propres préavis de grève, et la direction de la $\mathrm{SNCF}$, qui refuse de négocier avant la reprise du travail. L'intransigeance de la direction est soutenue par le gouvernement qui estime qu'il n'est pas question de céder aux syndicats alors que se prépare une nouvelle réforme des retraites. Cependant, malgré l'alliance contrainte avec Sud, le conflit n'embrase pas l'ensemble de la SNCF, notamment parce que des militants de Sud sont bien décidés à ne pas permettre à la CGT de restaurer son image de syndicat de lutte. Certes, des conflits se développent dans telle région ou tel établissement, mais la jonction entre les brasiers ne se fait pas (cela est toutefois moins vrai dans le sud de la France). De plus, l'effet d'entraînement sur les sympathisants des autres syndicats semble très faible à l'exception de l'Auvergne où la CFDT s'est cependant jointe au mouvement.

Le 13 avril, la direction fait un petit geste pour permettre à la CGT de sauver la face : elle refuse toujours des «négociations » avec les grévistes mais accepte des «discussions» au niveau des régions. Ces échanges donnent peu de résultats. En fait, la négociation collective est de plus en plus centralisée à la $\mathrm{SNCF}$ et les directeurs de région ne disposent guère de marge de manœuvre, ce qui, fondamentalement, traduit une recentralisation des modes de régulation en même temps que leur dilution à travers le développement d'une organisation matricielle et la quête d'une "performance sociale» (globale) de l'entreprise. Côté syndical, les deux organisations animatrices du conflit semblent avoir perdu confiance dans sa réussite et s'en remettent aux votes des assemblées générales pour sa poursuite éventuelle. De fait, le mouvement s'éteint petit à petit, les assemblées votant les unes après les autres sa suspension. Les conditions de déroulement du conflit, l'unité syndicale trop souvent en trompe-l'œil, les hésitations des cheminots (surtout sédentaires) à s'engager franchement et la détermination de la direction ont eu raison des grévistes.

Le «duopôle radical» sort battu du conflit mais en son sein, l'isolement de Sud est accentué par des déclarations gouvernementales qui, en revanche, 
épargnent la CGT ${ }^{(19)}$. Le mariage forcé entre la CGT et Sud n'est pas parvenu à construire un rapport de force suffisant pour faire reculer la direction. Et lorsque la CGT affirme qu'elle a finalement obtenu la création de 1300 à 2000 emplois, elle est démentie par les réformistes qui affirment qu'ils étaient acquis avant la grève. Pour la direction, ce dénouement marquerait un coup d'arrêt à la " gréviculture», selon l'expression de Louis Gallois reprise par ses successeurs, culture du conflit pour le conflit et de recherche de l'affrontement qui caractériserait l'entreprise. Ce conflit n'aurait-il servi à rien? De fait, les concessions de la direction sont peu tangibles. Mais qui pourrait affirmer que l'engagement pris par le gouvernement de renoncer à réduire les avantages relatifs des «régimes spéciaux» de retraites (déjà réformés en 2007) ne résulterait pas au moins en partie de cette mobilisation?

\section{Leçons d'un conflit : un continuum avec la négociation?}

La première caractéristique du conflit est de se dérouler dans un contexte de division-compétition intersyndicale. La CGT a d'abord vainement tenté de construire une alliance exclusive avec les «réformistes». Après leur retrait, on assiste à une «coopétition» (compétition et coopération) entre la CGT et Sud qui appellent à une grève reconductible. On peut ainsi parler de configurations au sens de Norbert Elias (1970, p. 157), assez évolutives et complexes (20). Pour ce qui est des relations entre la CGT et Sud, dans la deuxième phase du conflit, on peut repérer deux cas de figure : dans certains cas, les cheminots proches des deux syndicats se sont très bien entendus pour conduire la lutte de concert et les divisions entre organisations n'étaient pas plus significatives que les clivages internes à chacune d'elles. Dans une région comme le Nord où Sud est leader aux élections parmi les agents de conduite, les militants Sud n'étaient pas très motivés et, parfois, ont peu participé au mouvement. Ils ne voulaient pas faire le jeu de la CGT car ils estimaient qu'elle cherchait à restaurer son image aux yeux de ses propres adhérents, inquiets de l'alignement de la fédération CGT des cheminots sur la confédération CGT réputée moins radicale et actrice de la réforme «portant rénovation de la démocratie sociale». Les réticences des équipes Sud manifestées ici ou là, et confirmées par la fédération Sud-Rail, se sont

(19) Voir par exemple les déclarations de Christian Estrosi, ministre de l'Industrie, dépêche AFP du 21 avril 2010.

(20) Norbert Elias (1970, p. 157) illustre cette notion par l'image du jeu : «Quatre hommes assis autour d'une table pour jouer aux cartes forment une configuration. Leurs actes sont interdépendants [...]. Ce qu'il faut comprendre par configuration, c'est la figure globale toujours changeante que forment les joueurs; elle inclut non seulement leur intellect mais toute leur personne, les actions et les relations réciproques [...]. L'interdépendance des joueurs, condition nécessaire à l'existence d'une configuration spécifique, est une interdépendance d'alliés mais aussi d'adversaires.» attirées les foudres de la CGT ironisant sur ces révolutionnaires qui ne font pas grève (tract de la CGT-cheminots du Nord, 14 avril 2010). L'attitude de ces deux organisations, en rivalité pour occuper l'aile gauche de l'échiquier syndical (Sud) ou ne pas le délaisser (CGT), a donc engendré, selon les établissements, des résultats contrastés.

Ce conflit se caractérise aussi - deuxième trait par son caractère catégoriel, puisqu'il a essentiellement ciblé et mobilisé des «roulants » (conducteurs de train et contrôleurs). La courbe des taux de participation à la grève fournis par la direction (voir le graphique 3) montre même que c'est au deuxième jour du conflit, lorsque le taux de participation des roulants chute rapidement, que l'implication d'autres catégories monte brièvement en puissance avant de retomber ensuite.

\section{Graphique 3 : Taux de grévistes lors du conflit de la SNCF d'avril 2010 (en \% des personnels concernés)}

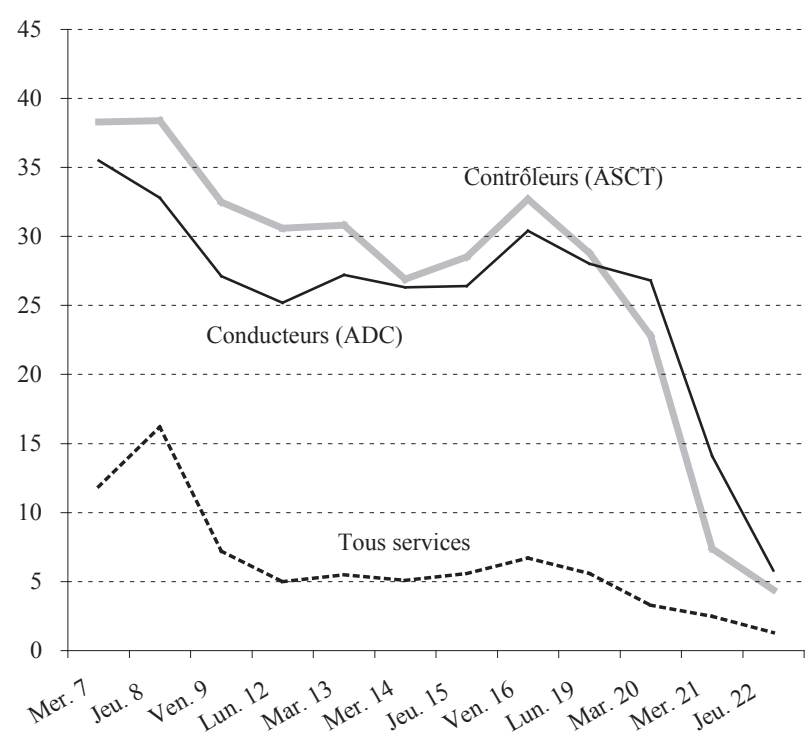

Source : SNCF, DRH. Graphique réalisé par nos soins.

De plus, depuis mars 2009, date de la mise en œuvre de la loi de 2008, les relations sociales à la SNCF paraissent avoir évolué. La CGT est en effet signataire des trois accords qui ont été validés : les accords des 11 janvier et 22 février 2010 (deux accords à cette dernière date) concernant le droit syndical (salariés «mis à disposition» des syndicats), la durée des mandats des représentants du personnel à élire en 2011 et le recours au vote électronique lors des scrutins professionnels. La négociation a porté sur une proposition de la direction qui, trouvant lourde et coûteuse l'organisation bisannuelle d'élections professionnelles, a concédé à la CGT des mandats de trois ans (alors qu'elle-même souhaitait quatre ans) en échange de l'acceptation d'une 
large expérimentation du vote électronique ${ }^{(21)}$. La signature de la CGT était essentielle pour valider ces accords car les deux autres organisations signataires - l'Unsa et la CFDT-FGAAC - ne disposaient pas d'une audience suffisante, comme on l'a déjà souligné, pour les valider. Sud, de son côté, était opposé à ce changement qu'il estimait susceptible d'éloigner les salariés des enjeux des relations professionnelles ${ }^{(22)}$.

Malgré ces trois accords, les rapports entre la direction et la CGT sont demeurés tendus début 2010 car la CGT a le sentiment de n'être pas suffisamment entendue. La mise en application de nouvelles règles induites par la loi n'a pas permis de changer la manière dont chaque acteur se représente l'autre partie. Puisque jusqu'alors, la loi n'a pas réussi à changer les modes de reconnaissance, c'est au conflit - pour reprendre l'approche classique de Georg Simmel (1995) - qu'a échu ce rôle.

Pourquoi cet échec alors même que les pouvoirs publics, en particulier l'exécutif, et des directions d'entreprise de différentes branches, ont par pragmatisme fait de la CGT un interlocuteur privilégié au moins jusqu'en 2009?

Tout d'abord, la fédération CGT des cheminots ne paraît pas majoritairement favorable à l'accentuation du «grand virage» (ANDOLFATto, LaBBÉ, 2009), conversion à un certain réalisme et à une démarche autonome pris par la confédération depuis 1999. On ne saurait toutefois exagérer ce qui serait un fossé entre stratégies fédérale et confédérale. En outre, un conflit social ne se réduit pas à des stratégies d'appareils. La fédération des cheminots CGT s'est aussi résolue à la grève parce que sa propre base s'inquiétait, à tort ou à raison, d'un certain immobilisme de l'appareil fédéral face aux restructurations et peutêtre aussi, du caractère infructueux de la négociation sur les salaires. Enfin, il ne faut pas sous-estimer la concurrence avec Sud-Rail qui a pu pousser la CGT à une sorte de grève "préemptive» - pour faire allusion à certaines analyses stratégiques (SMOUTS, Battistella, Vennesson, 2006) - en vue de conserver son leadership dans les luttes.

Enfin, s'agissant de la SNCF, on ne peut examiner la loi d'août 2008 indépendamment d'un «bouquet» de textes relatifs aux relations professionnelles,

(21) Selon un syndicaliste CGT, la question de la durée des mandats est en relation avec la crise des vocations syndicales. D'une part, des mandats trop longs découragent certains adhérents pressentis pour figurer sur les listes et tendent donc à rendre difficile leur constitution et, d'autre part, l'âge moyen des adhérents étant plutôt élevé, il est possible qu'ils n'aient pas le temps de terminer un mandat trop long avant leur départ à la retraite.

(22) En 2011, trois autres accords seront signés par la CGT : «convergence des primes de travail des sédentaires» (10/05/2011), «mise en place d'un groupe spécial de négociation en vue de la création d'un comité d'entreprise européen au sein du groupe $\mathrm{SNCF}$ » (12 juillet 2011), «emploi des travailleurs handicapés - années 2012 à 2015» (6 décembre 2011). notamment la loi du 21 août 2007 sur le «service minimum» dans les transports publics terrestres en cas de grève. L'objectif de ce texte est de prévenir la conflictualité en prévoyant des sas entre l'émergence d'un différend et l'éclatement de la grève. Cette loi permet aux syndicats représentatifs de déposer une demande de concertation immédiate (DCI) qui doit réunir les parties dans les cinq jours, passage obligé avant le dépôt d'un préavis de grève, qui lui-même ne prendra effet que cinq jours plus tard : il y a donc désormais dix jours de latence avant le déclenchement d'une grève. Cependant, dans le secteur des chemins de fer, les objectifs poursuivis sont loin d'avoir été atteints. Signe du caractère tendu des relations à la SNCF, un rapport de la Cour des comptes relève que la quantité de DCI a fortement cru en 2008, c'est-à-dire avant l'application de la loi sur la représentativité. Leur nombre, 2537 en 2008, a pratiquement triplé par rapport aux deux années précédentes, et «les trois quarts des préavis déposés se transforment en grève, ce qui représente un taux jamais atteint auparavant $\gg(23)$. Cependant il ne s'agissait là que d'un bilan «à chaud» de cette réforme dont les effets - sur le moyen terme restent à évaluer.

La réforme de 2008 a cherché à refonder la «représentativité au travail» en France, en substituant à un système descendant (règles de représentativité définies par des normes juridiques d'origines supérieures) un système ascendant où la représentativité est d'abord déterminée au niveau de l'entreprise. Au sens propre, il s'agit bien d'une refondation avec l'objectif de re-légitimer les organisations syndicales, de favoriser des organisations majoritaires et donc de limiter la fragmentation syndicale, ce qui permettrait d'inverser la courbe de syndicalisation car l'éclatement desservirait l'adhésion syndicale. La loi a aussi pour objectif de «dynamiser» ou de «pacifier» les relations professionnelles en tendant vers la notion d'accord majoritaire : ainsi, seules les organisations qui ont une audience importante peuvent signer des accords, ce qui devrait les conduire à davantage s'engager dans le jeu de la négociation.

Ce cadre «quasi-constitutionnel» appliqué aux relations professionnelles est finalement contraignant. Il est censé inverser le cours d'une histoire syndicale française marquée par le pluralisme, dépasser la tension entre un syndicalisme intégré et un syndicalisme de «contestation» et promouvoir une nouvelle «démocratie sociale», qui au demeurant reste à définir.

(23) SNCF : réformes sociales et rigidités de gestion, Cour des comptes, Rapport public annuel 2010, février 2010, p. 10. 


\section{Encadré 2}

\section{Élections professionnelles de 2011}

Le 24 mars 2011, les nouvelles élections professionnelles intervenues à la SNCF, sans bouleverser les rapports de force antérieurs entre les organisations professionnelles, permettaient aux «réformistes» (Unsa et CFDT-FGAAC) de franchir le seuil de $30 \%$ : les résultats de ces deux organisations s'établissent désormais à $35 \%$ des suffrages exprimés contre $29,9 \%$ auparavant. Ce gain s'est réalisé au détriment de l'audience des organisations animatrices du conflit de 2010 : la CGT (surtout) et, dans une moindre mesure, Sud (voir le graphique 1 supra et le tableau ci-dessous). Ce nouveau contexte ouvre le jeu social, offrant à la direction le choix de ses interlocuteurs.

Tableau : Résultats des élections aux comités d'entreprise de la SNCF en 2009 et 2011 (en \% des suffrages exprimés)

\begin{tabular}{|l|c|c|c|c|c|c|c|}
\hline & CGT & Unsa & $\begin{array}{c}\text { Sud- } \\
\text { Rail }\end{array}$ & $\begin{array}{c}\text { CFDT } \\
\text { (dont } \\
\text { FGAAC) }\end{array}$ & FO & CFTC & $\begin{array}{c}\text { CFE } \\
\text {-CGC }\end{array}$ \\
\hline $\mathbf{2 0 0 9}$ & 39,8 & 18,1 & 17,7 & 11,6 & 8,0 & 5,4 & - \\
\hline $\mathbf{2 0 1 1}$ & 37,4 & 21,5 & 17,4 & 13,8 & 8,6 & 1,0 & 0,4 \\
\hline
\end{tabular}

Source : SNCF, DRH.

Peut-on dire que les relations professionnelles à la SNCF ont été profondément modifiées par ce nouveau cadre? En fait, l'ancien contexte syndical et relationnel n'a pas disparu, les acteurs syndicaux n'ont pas été nécessairement renforcés tandis que plusieurs ont été fragilisés et la négociation collective n'a pas subitement changé de nature. Les organisations «réformistes» qui ont conservé leur «représentativité» ne parviennent pas à s'engager dans un processus de co-construction des normes qui marquerait l'avènement d'une ère nouvelle promue par la réforme, tandis que les syndicats «radicaux» ne le veulent ou ne le peuvent pas. $\mathrm{Au}$ contraire, sous les auspices de la loi d'août 2008, la SNCF a connu en 2010 l'un de ses plus importants conflits sociaux depuis plusieurs années. Cela illustre certes que «refoulé, le social finit toujours par ressurgir» (KaPlan, Minard, 2004), tout en soulignant les limites d'une réforme censée favoriser la «démocratie sociale». On pourrait même se demander si en bloquant ces relations, la loi n'a pas, au moins provisoirement, aggravé la situation aux yeux de ceux qui appelaient de leurs vœux des relations sociales moins tendues. En outre, à la SNCF, le calcul de la représentativité nationale sur la base des élections dans les régions complexifie les relations professionnelles puisqu'une organisation peut être représentative au niveau national mais ne pas l'être au niveau régional ou inversement (voir tableau en annexe). Cet entrelacs de situations existe aussi dans d'autres entreprises comme par exemple à Pôle emploi.
Cependant, la combinaison de ces facteurs de «crise» n'est pas nécessairement durable et peut aussi s'envisager comme une étape dans un processus de transformation plus long. De fait, les résultats des élections professionnelles de mars 2011 devraient permettre de débloquer - au moins partiellement - cette situation (voir encadré 2). Enfin, la loi a apporté un certain nombre de changements. Le nombre des organisations représentatives a été réduit, même si la direction doit encore compter avec quatre interlocuteurs et même plus dans certains établissements. La loi a également déstabilisé les syndicats en les obligeant à se remettre en cause et à réajuster leur stratégie pour demeurer dans le jeu. Plus fondamentalement, elle pourrait constituer une ressource pour la CGT, qui souhaiterait jouer un rôle plus actif dans la négociation. Or elle est handicapée par des relations parfois tendues avec sa base, mais aussi, en externe, avec Sud-Rail, qui exerce une valence positive sur une partie de ses adhérents. Par les effets de seuil, la loi a également eu au moins temporairement pour résultat d'affaiblir un "pôle réformiste» sur lequel la direction n'a pu réellement s'appuyer pour signer des accords. Cette faiblesse, redoublée par les ambivalences de la CGT, a finalement rendu les relations professionnelles provisoirement plus difficiles. Il est vrai toutefois que les restructurations présentes et futures à la SNCF sont si profondes qu'on ne voit pas comment elles pourraient se dérouler sans heurt, sans déception et remise en cause des acteurs. 


\section{Bibliographie}

Andolfatto D., Dressen M., Finez J. (2012), «Conflictualité cheminote en France au tournant des $\mathrm{XX}^{\mathrm{e}}$ et $\mathrm{XXI}^{\mathrm{e}}$ siècles. Comparer les conflits pour les comprendre», Organisations, professions, marchés et cultures dans les chemins de fer, Second ISA Forum of sociology, Social justice and democratization, Buenos Aires (Argentina), August 1-4, 2012, 19 p.

Andolfatto D., LabBé D. (2009), Toujours moins. Déclin du syndicalisme à la française, Paris, Gallimard.

Andolfatto D., LabBÉ D. (dir.) (2007), Les syndiqués en France, Rueil-Malmaison, Liaisons/Kluwer.

Bevort A. (2008), «De la position commune sur la représentativité au projet de loi : renouveau et continuité du modèle social français», Droit social, $\mathrm{n}^{\circ}$ 7-8, pp. 823-833.

Bors P. (1971), Paysans de l'Ouest. Des structures économiques et sociales aux options politiques depuis l'époque révolutionnaire dans la Sarthe, Paris, Flammarion.

Cohen V., Dressen M. (2011), «Pénibilité et violence morale chez les conducteurs de trains. Opérateurs privés et opérateur public», in Dressen M., Durand J.-P. (dir.), La violence au travail, Toulouse, Octares.

Contamin J.-G., Delacroix R. (2009), «Les transformations des formes d'engagement au prisme du local : l'exemple de la CGT du Nord», Politix, vol. 22, $\mathrm{n}^{\circ} 85$, pp. 81-104.

Dubois P. (1974), Mort de l'État-patron, Paris, Éditions économie et humanisme / Les éditions ouvrières, coll. «relations sociales $»$.

Dubreuil F., Basilien J.-P. (2012), «De la régulation sociale à la performance sociale. Réguler une conflictualité qui se déplace, se transforme, se déguise...», Entreprise \& Personnel, Étude n 302.
Duriez B., SAwicki F. (2003), «Réseaux de sociabilité et adhésion syndicale. Le cas de la CFDT», Politix, vol. 16, $\mathrm{n}^{\circ} 63$, pp. 17-51.

Elias N. (1970), Qu'est-ce que la sociologie?, Paris, Pocket, (éd. 2003).

Hirschman A. (1970), Exit, voice, loyalty. Responses to decline in firms, organizations and states, Cambridge, Harvard University Press.

Jobert B. (1996), «Actualité des corporatismes», Pouvoirs ${ }^{\circ} 79$, pp. 21-34.

Kaplan S.L., Minard P. (dir.) (2004), La France malade du corporatisme?, Paris, Belin.

Mabileau A. (1991), Le système local en France, Paris, Montchrestien.

Ribeill G. (1994), «SNCF : du malaise social à la cassure corporative», Travail, $n^{\circ} 31$, pp. 37-62.

Rosanvallon P. (1987), La question syndicale, Paris, Calmann-Lévy.

Simmel G. (1995), Le Conflit, Paris, Circé.

Smouts M.-C., Battistella D., Vennesson P. (2006), Dictionnaire des relations internationales : approches, concepts, doctrines, Paris, Dalloz, (2e éd.).

TEXIER P.-Y. (1998), «Un impossible compromis social français» in Auvergnon P. et al., L'État à l'épreuve du social, Paris, Syllepse, pp. 230-241.

Tournadre-Plance J., Verrier B. (2008), «La démocratie politique représentative comme modèle pour la démocratie sociale?», La note de veille, $\mathrm{n}^{\circ} 99$, Centre d'analyse stratégique.

VACQUin H. (2009), Le conflit de Paris-Saint-Lazare, rapport pour la direction de la SNCF. 


\section{Annexe}

Les distorsions entre la représentativité au niveau national et au niveau des comités d'établissement en 2009 et 2011

\begin{tabular}{|c|c|c|c|c|c|c|c|c|}
\hline & \multicolumn{5}{|c|}{ Représentativité } & \multirow{2}{*}{\multicolumn{3}{|c|}{$\begin{array}{c}\text { Majorité qualifiée de } 30 \% \\
\text { pour signer seul des accords } \\
\text { (en nombre de comités d'entreprises) }\end{array}$}} \\
\hline & \multicolumn{2}{|c|}{$\begin{array}{c}\text { Nationale } \\
\text { (seuil des } 10 \% \text { atteint) }\end{array}$} & \multicolumn{3}{|c|}{$\begin{array}{c}\text { Sur } 27 \text { établissements } \\
\text { (seuil des } 10 \% \text { atteint) }\end{array}$} & & & \\
\hline & 2009 & 2011 & 2009 & 2011 & Différence & 2009 & 2011 & Différence \\
\hline CGT & oui & oui & 27 & 27 & 0 & 22 & 22 & 0 \\
\hline Unsa & oui & oui & 27 & 27 & 0 & 0 & 2 & +2 \\
\hline Sud & oui & oui & 22 & 22 & 0 & 2 & 3 & +1 \\
\hline $\begin{array}{l}\text { CFDT- } \\
\text { FGAAC }\end{array}$ & oui & oui & 15 & 22 & +7 & 0 & 0 & 0 \\
\hline $\begin{array}{l}\text { FO } \\
+ \text { CFE-CGC }\end{array}$ & non & non & 7 & $8^{(*)}$ & +1 & 0 & 0 & 0 \\
\hline CFTC & non & non & 5 & 0 & -5 & 0 & 0 & 0 \\
\hline
\end{tabular}

Source : SNCF, DRH. Tableau réalisé par nos soins.

(*) : Selon les CE, FO s'est ou non alliée avec la CFE-CGC et la CFTC

Ce tableau confirme la distorsion qui existe entre la représentativité nationale et la représentativité au niveau des comités d'établissement. La liste des organisations représentatives au niveau national ne change pas en 2011 par rapport à 2009. Le tableau montre en revanche le caractère partiellement instable des situations au niveau des comités d'établissement, du fait d'une composition évolutive des listes d'une élection à l'autre (cas de FO et de la CFE-CGC unies en 2011 et, ici ou là, alliées avec la CFTC). En outre, une organisation peut perdre

ou gagner en influence. Ainsi, en 2011, la progression de la CFDT-FGAAC est nette. Si l'on retient comme critère les organisations qui ont réuni $30 \%$ des suffrages exprimés au niveau des comités d'établissement, seuil qui leur permet de signer un accord sans être obligée de chercher des alliances, 1'Unsa et Sud-Rail progressent, ce qui constitue également une nouvelle donne qui n'a pas nécessairement d'effet direct sur les relations professionnelles au niveau national mais en a évidemment au niveau des établissements. 